\title{
Real Time Recognition of Handwritten Devnagari Signatures without Segmentation Using Artificial Neural Network
}

\author{
Shailendra Kumar Dewangan \\ Assistant Professor, Department of Electronics \& Instrumentation Engg \\ Chhatrapati Shivaji Institute of Technology, Durg, Chhattisgarh, India
}

\begin{abstract}
Handwritten signatures are the most commonly used method for authentication of a person as compared to other biometric authentication methods. For this purpose Neural Networks (NN) can be applied in the process of verification of handwritten signatures that are electronically captured. This paper presents a real time or online method for recognition and verification handwritten signatures by using $\mathrm{NN}$ architecture. Various features of signature such as height, length, slant, Hu's moments etc are extracted and used for training of the NN. The objective of online signature verification is to decide, whether a signature originates from a given signer. This recognition and verification process is based on the instant signature image obtained from the genuine signer and a few images of the original signatures which are already part reference database. The process of Devnagari signature verification can be divided it into sub-processes as pre-processing, feature extraction, feature matching, feature comparison and classification. This stepwise analysis allows us to gain a better control over the precision of different components.
\end{abstract}

Index Terms - Artificial Neural Networks (ANN), Handwritten Signature Verification (HSV), Hu's moment invariants, Real time recognition method, Signature Recognition

\section{INTRODUCTION}

Development of a signature recognition system for Devnagari is difficult because there are compound character shapes in the script and the characters in words are topologically connected. Here our focus is on the recognition of online handwritten Devnagari (Hindi) signatures that can be used in common applications like bank cheques, commercial forms, government records, bill processing systems, post code recognition, signature verification, passport readers, online document recognition generated by the expanding technological society etc. Handwriting has continued to persist as a means of communication and recording information in day-to-day life even with the introduction of new technologies [1]. Challenges in handwritten signatures recognition lie in the variation and distortion of handwritten signatures, since different people may use different style of handwriting and direction to draw the same shape of any Devnagari character [2]. This overview describes the nature of handwritten language, how it is translated into electronic data, and the basic concepts behind written language recognition algorithms.

Handwritten Devnagari signatures are imprecise in nature as their corners are not always sharp, lines are not perfectly straight, and curves are not necessarily smooth, unlikely the printed signature. Furthermore, Devnagari character can be drawn in different sizes and orientation in contrast to handwriting which is often assumed to be written on a baseline in an upright position [3]. Therefore, a robust online Devnagari handwritten signature recognition system has to account for all of these factors. An approach using Artificial Neural Network is considered for recognition of Handwritten Devnagari Signatures.

In this paper a method is proposed for recognition of Devnagari handwritten signature. Novelty of this proposed work is that there is no requirement of segmentation. Under Section - 1 proposed work is introduced. It also deals with objective and motivation of this work. Then after previous work done related to this theme and architecture of this system are also discussed briefly. In Section - 2 methodology is discussed along with brief description of performance parameters used for obtaining the result. Section - 5 gives result analysis and discussions. Finally Section - 6 brief conclusion and scope for further work.

\section{A. Proposed Work}

In this proposed work which requires the sample database of handwritten Devnagari signatures, observes various features of the signature sample. On basis of its comparison with the signature samples available in the database, result is shown about authenticity of the signature sample. We can apply this algorithm on different types of applications where human authentication is required.

\section{B. Objective}

The objective of this work is to design a system for recognition of handwritten signature in Devnagari language. Our first goal is to find the method or function which gives the maximum accuracy for recognizing the signature, when it is arbitrary selected and compared with the signature database. For these types of images it 
is too much difficult to choose such a function which can be the best for the process of feature extraction and comparison for standardization.

\section{Motivation}

The motivation behind the project is the growing need for a full proof signature verification scheme which can guarantee maximum possible security against fake signatures. The prospect of minimizing the memory required for storing the extracted feature points has also been a driving force in the commencement of this project.

\section{Previous Work}

Devnagari script recognition is very challenging due to the complex structural properties of the script. Two different methods for extracting features from handwritten Devnagari characters can be used are the Curvelet Transform and the Character Geometry [1]. Topographical features of strokes visible with respect to views from different directions which can be successfully used for recognition of face, handwritten signature and printed characters [2]. Three feature extraction techniques as Hu's Moment Invariants, Zernike moments and Krawtchouk moment can be used for recognition of signatures. These methods provide the stastical data which can be tested for various parameters like the length of feature vector, redundancy and accuracy [3]. A Neural Network based on-line signature verification system can be designed by using a set of feature vectors that can represent the characters of the signatures. By forming and verification of feather sets false acceptance (FA) and false rejection (FR) rate can be reduced [4].

A writer independent offline handwritten signature verification system based on grey level feature extraction and Real Adaboost algorithm can be designed by using both global and local features of the script [5]. Object recognition can also be successfully done by using methods based on image moments. Various types of moments (geometric moments, complex moments) and moment-based invariants with respect to various image degradations and distortions (rotation, scaling, affine transform, image blurring, etc.) can be used as shape descriptors for classification [6]. Also efficient numerical algorithms can be used for moment computation and demonstrate practical examples of using moment invariants in real applications [7].

\section{E. Architecture of Recognition System}

The entire process of recognition of handwritten signature can be categorized six sections. The architecture of the signature recognition system is shown in Fig. 1.

(a) The first section is to obtain sample signature image from the signer. This sample image is later on compared with the database which is generated by collecting genuine signatures, collected from various human subjects. (b) In the second section the loaded sample signature image is cropped, because further testing process is done on that cropped section only. Cropping refers to the process of removing unwanted areas from a photographic or illustrated image.

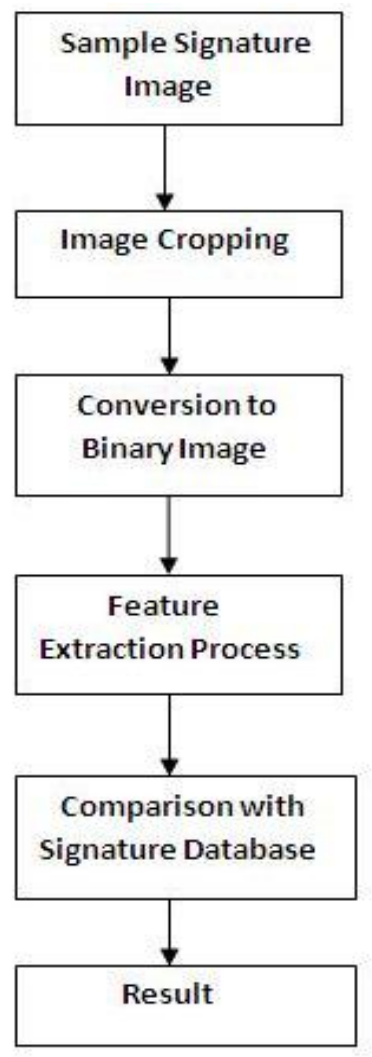

Fig. 1 : Architecture of Signature Recognition System

(c) In the third section the cropped image is converted into binary image. A binary image is a digital image that has only two possible values for each pixel. Typically the two colors used for a binary image are black and white though any two colors can be used.

(d) The fourth section all the specified features of sample signature are extracted. Feature extraction involves simplifying the amount of resources required to describe a large set of data accurately. When performing analysis of complex data one of the major problems stems from the number of variables involved.

(e) The fifth section all the features of sample signature image are compared with the signature database.

(f) On basis of this comparison it is decided that whether the sample signature belongs to the signature database or it is an authorized signature.

\section{F. Designing Process}

The entire designing process can be subdivided in two steps which are described as followings.

\section{a) System Design Process :}

In order to make a project which can be applicable for real world situation, we are required to do really 
brainstorming. First thing of discussion are that how many signature image signals are taken as an input signal for generalizing the features of that sample signature for our study and also it is important to decide that in which format the images should be taken? Here we have collected 10 samples of signatures of each person.

\section{b) Program Design :}

In our program we have made software using MATLAB programming for analysis of Devnagari handwritten signature using moment invariant analysis . The first step of the process was the acquisition of the features of the particular signature sample, then after according to the available features a threshold value is obtained and it is compared with the database of sample signatures. After comparison the signature sample is identified as genuine or unauthorized one.

\section{METHODOLOGY}

This section describes the methodology behind the system development. It discusses the pre-processing performed, the signature database, and the NN features. Signature Recognition Systems need to preprocess the data [4] [5]. It includes a series of operations to get the results. The major steps are as follows -

\section{A. Data Acquisition :}

For recognition purpose at the first stage the signatures are required to be processed by the system. Therefore they should be in the digital image format. We need to obtain the instant sample signature image from the signer for the verification purpose. For this purpose devices such as digital tablets can be used shown in Fig. 2. Data acquisition process is a process where the real time inputs of signature from the digitizing tablet and the special pen are read into the CPU for processing and to store the signature in to the database. The digitizing tablet is sending the real time inputs to the CPU for further processing and storage.

\section{B. Signature Pre-processing :}

The signature samples are required to be normalized and resized up to proper dimensions. Then after the background noise is removed and thinning of the signature sample is performed. This yields a signature template which can be used for extracting the features. Therefore minimal signature pre-processing is required.

\section{Feature Extraction :}

The features extracted from signatures or handwriting play a vital role in the success of any feature based HSV system. If a poorly constructed feature set is used with little insight into the writer's natural style, then no amount of modeling or analysis is going to result in a successful system. Further, it is necessary to have multiple, meaningful features in the input vector to guarantee useful learning by the NN. In this paper we have considered the most important feature under our consideration for the process of signature verification, is Hu's Moment Invariants.

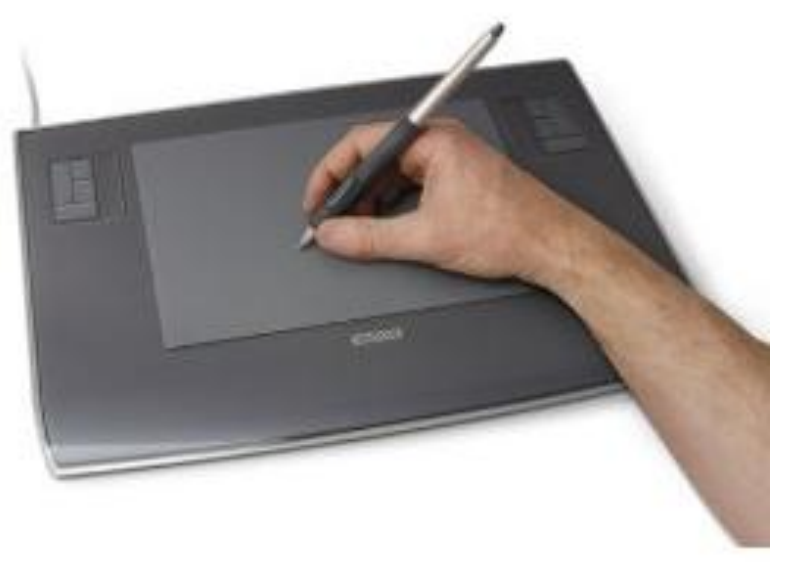

Fig. 2 : Digital Tablet

\section{Hu's Moment Invariants :}

In image processing, computer vision and related fields image moments are very useful for image analysis. $\mathrm{Hu}$ derived these expressions from algebraic invariants applied to the moment generating function under a rotation transformation [6]. They consist of groups of nonlinear centralized moment expressions. The result is a set of absolute orthogonal (i.e. rotation) moment invariants, which can be used for scale, position, and rotation invariant pattern identification. Simple properties of images which are found via image moments include area, total intensity, centroid and information about its orientation. They are computed from normalized centralized moments up to order three $\mathrm{I}_{\mathrm{n}}$ and $\mathrm{n}^{\text {th }} \mathrm{Hu}$ invariant moment. Here mean values of $\mathrm{x}$ and $y$ are represented by equation 2.1 and 2.2 ,

$$
\begin{aligned}
& \bar{x}=\frac{M_{10}}{M_{00}} \\
& \bar{y}=\frac{M_{01}}{M_{00}}
\end{aligned}
$$

The central moments can be represented by following equation 2.3 and 2.4 ,

$$
\begin{aligned}
& \mu_{p q}=\int_{-\infty}^{\infty} \int_{-\infty}^{\infty}(x-\bar{x})^{p}(y-\bar{y})^{q} f(x, y) d x d y \\
& \mu_{p q}=\sum_{m}^{p} \sum_{n}^{q}\left(\begin{array}{l}
p \\
m
\end{array}\right)\left(\begin{array}{l}
q \\
n
\end{array}\right)(-\bar{x})^{(p-m)}(-\bar{y})^{(q-n)} M_{m n}
\end{aligned}
$$


If $\mathrm{f}(\mathrm{x}, \mathrm{y})$ is a digital image, then the previous equation becomes,

$\mu_{p q}=\sum_{x} \sum_{y}(x-\bar{x})^{p}(y-\bar{y})^{q} f(x, y)$

The central moments of order up to 3 are:

$$
\begin{aligned}
& \mu_{00}=M_{00} \\
& \mu_{10}=0 \\
& \mu_{11}=M_{11}-\bar{x} M_{01}=M_{11}-\bar{y} M_{10} \\
& \mu_{20}=M_{20}-\bar{x} M_{10} \\
& \mu_{02}=M_{02}-\bar{y} M_{01} \\
& \mu_{21}=M_{21}-2 \bar{x} M_{11}-\bar{y} M_{20}+ \\
& 2 \bar{x}^{2} M_{01} \\
& \mu_{12}=M_{12}-2 \bar{y} M_{11}-\bar{x} M_{02}+2 \bar{y}^{2} M_{10} \\
& \mu_{30}=M_{30}-3 \bar{x} M_{20}+2 \bar{x}^{2} M_{10}
\end{aligned}
$$

$$
\mu_{03}=M_{03}-3 \bar{y} M_{02}+2 \bar{y}^{2} M_{01}
$$

Central moments are translational invariant. Information about image orientation can be derived by first using the second order central moments to construct a covariance matrix. Rotational invariants moment equations are given as below :

$$
\begin{aligned}
& \mu_{20}^{\sigma}=\frac{\mu_{20}}{\mu_{00}}=\frac{M_{20}}{M_{00}}-\bar{x}^{2} \\
& \mu_{02}^{\sigma}=\frac{\mu_{02}}{\mu_{00}}=\frac{M_{02}}{M_{00}}-\bar{y}^{2} \\
& \mu_{11}^{\sigma}=\frac{\mu_{11}}{\mu_{00}}=\frac{M_{11}}{M_{00}}-\bar{x} \bar{y}
\end{aligned}
$$

It is possible to calculate moments which are invariant under translation, changes in scale, and also rotation. Most frequently used are the $\mathrm{Hu}$ set of invariant moments are :

$$
\begin{aligned}
& M_{1}=\eta_{20}+\eta_{02}, \\
& M_{2}=\left(\eta_{20}-\eta_{02}\right)^{2}+4 \eta_{11}^{2}, \\
& M_{3}=\left(\eta_{30}-3 \eta_{12}\right)^{2}+\left(3 \eta_{21}-\eta_{03}\right)^{2}, \\
& M_{4}=\left(\eta_{30}+\eta_{12}\right)^{2}+\left(\eta_{21}+\eta_{03}\right)^{2},
\end{aligned}
$$

$\mathbf{M}_{5}=\left(\eta_{30}-3 \eta_{12}\right)\left(\eta_{12}+\eta_{30}\right)\left[\left(\eta_{12}+\eta_{30}\right)^{2}-3\left(\eta_{21}+\right.\right.$ $\left.\left.\eta_{03}\right)^{2}\right]+\left(3 \eta_{21}-\eta_{03}\right)\left(\eta_{21}+\eta_{03}\right)\left[3\left(\eta_{30}+\eta_{12}\right)^{2}-\left(\eta_{21}+\right.\right.$ $\left.\left.\eta_{03}\right)^{2}\right]$

$\mathbf{M}_{6}=\left(\eta_{20}-\eta_{02}\right)\left[\left(\eta_{30}+\eta_{12}\right)^{2}-\left(\eta_{21}+\eta_{03}\right)^{2}\right]+4 \eta_{11}\left(\eta_{30}+\right.$ $\left.\eta_{12}\right)\left(\eta_{21}+\eta_{03}\right)$,

$\mathrm{M}_{7}=\left(3 \eta_{21}-\eta_{03}\right)\left(\eta_{30}+\eta_{12}\right)\left[\left(\eta_{12}+\eta_{30}\right)^{2}-3\left(\eta_{21}+\right.\right.$ $\left.\left.\eta_{03}\right)^{2}\right]+\left(3 \eta_{12}-\eta_{30}\right)\left(\eta_{21}+\eta_{03}\right)\left[3\left(\eta_{30}+\eta_{12}\right)^{2}-\left(\eta_{21}+\right.\right.$ $\left.\left.\eta_{03}\right)^{2}\right]$,

The first one, $\mathbf{M}_{1}$, is analogous to the moment of inertia around the image's centroid, where the pixels' intensities are analogous to physical density. The last one, $\mathbf{M}_{7}$, is skew invariant, which enables it to distinguish mirror images of otherwise identical images. A general theory on deriving complete and independent sets of rotation invariant moments was proposed by $\mathbf{J}$. Flusser [7] and T. Suk [8]. They showed that the traditional Hu's invariant set is neither independent nor complete. $\mathrm{M}_{2}$ and $\mathrm{M}_{3}$ are not very useful for pattern recognition, as they are dependent. On the original Hu's set of moment invariants there is a missing third order independent moment invariant which given by following equation:

$\mathbf{M}_{8}=\eta_{11}\left[\left(\eta_{30}-\eta_{12}\right)^{2}-\left(\eta_{03}+\eta_{21}\right)^{2}\right]-\left(\eta_{20}-\eta_{02}\right)\left(\eta_{30}+\right.$ $\left.\eta_{12}\right)\left(\eta_{03}+\eta_{21}\right)$

\section{E. Performance Parameters :}

For ensuring good performance for signature recognition system few parameters are required to be set. Such performance parameters for our Devnagari handwritten signature recognition system are discussed below :

a) False Accept Rate or False Match Rate (FAR or FMR) :

The false acceptance rate is given by the number of fake signatures accepted by the system with respect to the total number of comparisons made. It is the probability that the system incorrectly matches the input pattern to a non-matching template in the database. It measures the percent of invalid inputs which are incorrectly accepted.

b) False Reject Rate or False Non-Match Rate (FRR or FNMR) :

The false rejection rate is the total number of genuine signatures rejected by the system with respect to the total number of comparisons made. Both FAR and FRR depend on the threshold variance parameter taken to decide the genuineness of an image. If we choose a high threshold variance then the FRR is reduced, but at the same time the FAR also increases. If we choose a low threshold variance then the FAR is reduced, but at the same time the FRR also increases. The FRR is the measurement of the probability that a biometric system will fail to identify an individual who is properly enrolled. It measures the percent of valid inputs which are incorrectly rejected. 
c) Receiver Operating Characteristic or Relative Operating Characteristic (ROC) :

The ROC plot is a visual characterization of the tradeoff between the FAR and the FRR. In general, the matching algorithm performs a decision based on a threshold which determines how close to a template the input needs to be for it to be considered a match. If the threshold is reduced, there will be less false nonmatches but more false accepts. Correspondingly, a higher threshold will reduce the FAR but increase the FRR. This more linear graph illuminates the differences for higher performances.

\section{d) 2.5.4 Equal Error Rate or Crossover Error Rate (EER or CER) :}

The rates at which both accept and reject errors are equal. The value of the EER can be easily obtained from the ROC curve. The EER is a quick way to compare the accuracy of devices with different ROC curves. In general, the device with the lowest EER is most accurate.

\section{EXPERIMENTAL SET - UP}

We have been concerned here with evaluating moment invariants as a feature space in a pattern recognition problem, predicting how they will behave given the classes and noise conditions of the problem. For this purpose, we accumulated enough background to help understand the nature of moment invariants and derived basic performance results in terms of the classes and noise.

\section{A. Training and Testing}

A total of 500 genuine signatures were collected from a population of 50 human subjects which included 22 women and 28 men. Out of which seven of them are left handed writers. Our main task is to recognizing the signature with good feature recognition techniques which provide good results on the signature recognition dataset. We train the system using a training set of signature obtained from a person. Designing of a classifier is a separate area of research. The decision thresholds required for the classification are calculated by considering the variation of features among the training set. Separate set of thresholds (user Specific) is calculated for each person enrolled, some system also use common threshold form all users.

For training and testing general fuzzy minimum maximum (FMM) neural network is used in our work. Fuzzy networks are useful for clustering (unsupervised) and classification (supervised) problems. Application of FMM is an attempt to improve classification performance of the recognition system. By using FMM training the total time required to train the database was achieved less than $173.77 \mu$ s and the time taken to recognize a signature sample was less than $125.67 \mathrm{~ms}$.

\section{B. Reason for using Hu's Moment Invariants}

This work is based on analysis of values of Hu's seven moment invariants. They consist of groups of nonlinear centralized moment expressions. The result is a set of absolute orthogonal (i.e. rotation) moment invariants, which can be used for scale, position, and rotation invariant pattern identification. These can be efficiently used in a simple pattern recognition application for successfully identifying various patterns [9]. The first one, $M_{1}$, is analogous to the moment of inertia around the image's centroid, where the pixels' intensities are analogous to physical density. The last one, $\mathbf{M}_{7}$, is skew invariant, which enables it to distinguish mirror images of otherwise identical images.

We have done analysis of fluctuation in moment invariant values of the same image for different positions. We took the original test image, scaled by one half of the test image and mirrored test image. For analyzing rotation effect we have taken rotated test image by $45^{\circ}, 90^{\circ}$ and $180^{\circ}$. These images are shown in Fig. 3 under (a) Original Test Image, (b) Scaled by one half, (c) Rotated by $45^{\circ}$, (d) Rotated by $90^{\circ}$, (e) Rotated by $180^{\circ}$, (f) Mirrored Image.

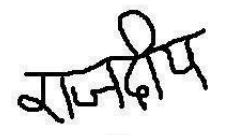

(a)

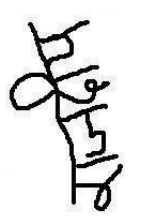

(c)

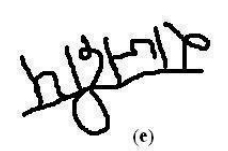

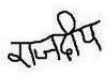

(b)

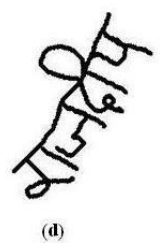

(d)

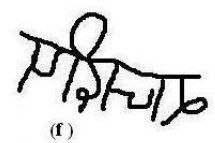

Fig. 3 : Different images for analysis

The moment values were observed for these six differently scaled and rotated images of the same signature image. The experimental values for these six images are shown in Table 3.1. 
Using Artificial Neural Network

Table 3.1 : Closeness of Moment Invariants values for Different Images

\begin{tabular}{|c|r|r|r|r|r|r|}
\hline $\begin{array}{c}\text { Moment } \\
\text { Invariant }\end{array}$ & \multicolumn{1}{|c|}{$\begin{array}{c}\text { Original } \\
\text { Image }\end{array}$} & \multicolumn{1}{c|}{$\begin{array}{c}\text { Half Size } \\
\text { Image }\end{array}$} & \multicolumn{1}{c|}{$\begin{array}{c}\text { Mirrored } \\
\text { Image }\end{array}$} & \multicolumn{1}{c|}{$\begin{array}{c}\text { Rotated } \\
\text { Image } \\
\left(\mathrm{By} 45^{0}\right)\end{array}$} & \multicolumn{1}{c|}{$\begin{array}{c}\text { Rotated } \\
\text { Image } \\
\left(\mathrm{By} 90^{0}\right)\end{array}$} & $\begin{array}{c}\text { Rotated Image } \\
\left(\mathrm{By} 180^{0}\right)\end{array}$ \\
\hline $\mathrm{M}_{1}$ & 0.039610 & 0.039622 & 0.039610 & 0.039610 & 0.039619 & 0.039610 \\
\hline $\mathrm{M}_{2}$ & 0.869102 & 0.869235 & 0.869102 & 0.869102 & 0.869131 & 0.869102 \\
\hline $\mathrm{M}_{3}$ & 0.004258 & 0.004267 & 0.004258 & 0.004258 & 0.0042576 & 0.004258 \\
\hline $\mathrm{M}_{4}$ & 0.112366 & 0.112456 & 0.112366 & 0.112366 & 0.112354 & 0.112366 \\
\hline $\mathrm{M}_{5}$ & -19.791654 & -19.792761 & -19.791654 & -19.791654 & -19.791782 & -19.791654 \\
\hline $\mathrm{M}_{6}$ & -2.392429 & -2.392531 & -2.392429 & -2.392429 & -2.392326 & -2.392429 \\
\hline $\mathrm{M}_{7}$ & -6.902151 & -6.902237 & 6.902151 & -6.902151 & -6.902435 & -6.902151 \\
\hline
\end{tabular}

We found the closeness of the values of the moments, which is independent of translation, scale change, mirroring and rotation (as shown in Table 3.1). There is only difference in sign of $\mathrm{M}_{7}$, which is for the mirrored image. This property can be used to detect whether the image is a mirrored image. This was the reason behind using Hu's moment invariants for this work.

\section{Results and Discussions}

After process of training the signature database we started testing each sample signature. On basis of recognition and rejection the experimental results regarding FRR, FAR and AER are shown in Table 3.2. These results show that with the increase of similarity false reject rate (FRR) the false acceptance rate (FAR) is decreased. The results of our examination show that in this method, the best value for the percentage of signature similarity is nearly $31 \%$. In this point we obtain the minimum error rate $($ MinErrRate $=$ min $(F A R$, FRR)). If we consider Average Error Rate (AER) are as following:

$\mathrm{AER}=(\mathrm{FAR}+\mathrm{FRR}) / 2$

Table 3.2 : Variation of FAR and FRR with the threshold Variance

\begin{tabular}{|c|c|c|c|c|c|c|}
\hline S. No. & $\begin{array}{c}\text { Threshold } \\
\text { Variance }\end{array}$ & $\begin{array}{c}\text { Nonuine } \\
\text { signatures } \\
\text { rejected (out of } \\
50)\end{array}$ & FRR & $\begin{array}{c}\text { No. of fake } \\
\text { signatures } \\
\text { accepted (out of } \\
50)\end{array}$ & FAR & $\begin{array}{c}\text { AER }= \\
\text { (FRR + } \\
\text { FAR)/2 }\end{array}$ \\
\hline 1. & 0.16 & 7 & $14.0 \%$ & 19 & $38.0 \%$ & $26.0 \%$ \\
\hline 2. & 0.14 & 12 & $24.0 \%$ & 17 & $34.0 \%$ & $34.0 \%$ \\
\hline 3. & 0.12 & 16 & $32.0 \%$ & 15 & $30.0 \%$ & $31.0 \%$ \\
\hline 4. & 0.10 & 20 & $40.0 \%$ & 12 & $24.0 \%$ & $34.0 \%$ \\
\hline 5. & 0.08 & 24 & $48.0 \%$ & 9 & $18.0 \%$ & $33.0 \%$ \\
\hline 6. & 0.06 & 28 & $56.0 \%$ & 7 & $14.0 \%$ & $35.0 \%$ \\
\hline 7. & 0.04 & 32 & $64.0 \%$ & 4 & $8.0 \%$ & $36.0 \%$ \\
\hline
\end{tabular}


Average Error Rate (AER) will be the smallest amount in $\mathrm{SS} \in[25,35]$. On the other hand, we have the best performance of the system in $(25 \% \leq \mathrm{SS} \leq$ $35 \%$ ). In this interval, we have the minimum value for Average Error Rate (AER) (shown in Fig. 4).

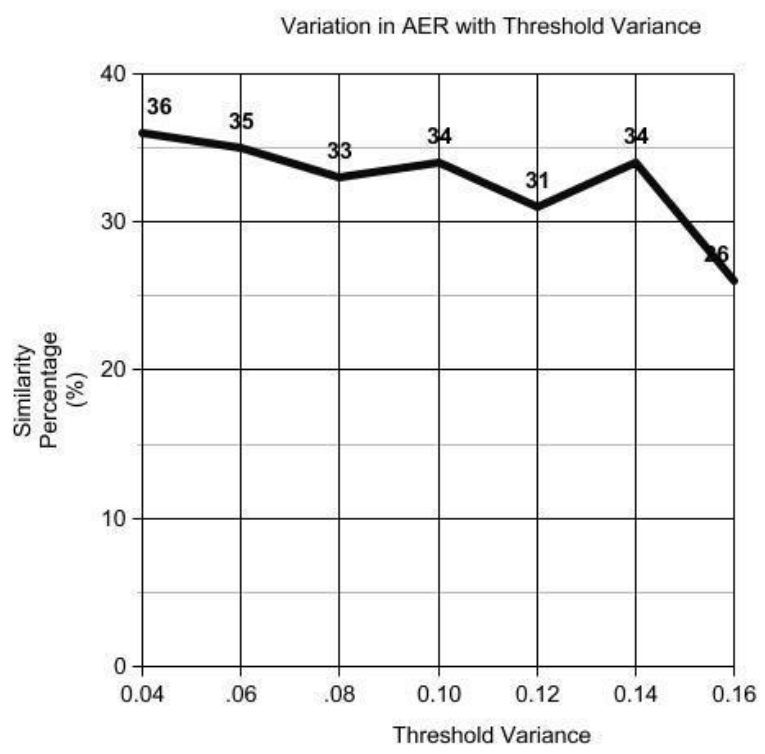

Fig. 4 : Performance of HDSR system

The minimum value of Average Error Rate (AER) for our work is achieved to be $33.0 \%$. Where the value of the FAR and the FRR meet one another, the point is called Equal Error Rate (EER) as it is shown in Fig. 5.

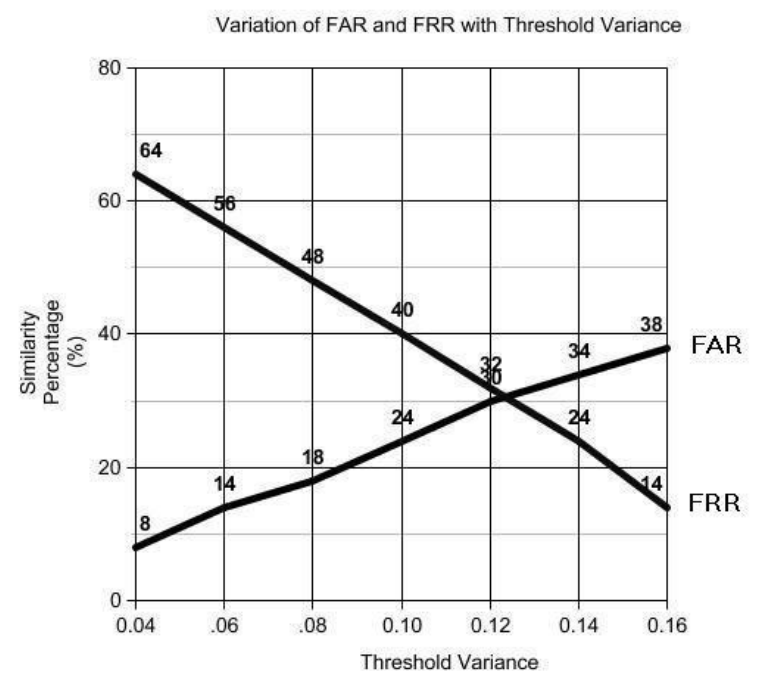

Fig. 5 : Signature Similarity Percentage

As a matter of fact, getting the best performance, we should consider $25 \% \leq \mathrm{SS} \leq 35 \%$. The fundamental result of this study is obtaining the average of minimum errors not in the maximum surface similarity. In other words, if the correctness of a signature is its high similarity to the original one, the correct signatures will be rejected because of minor differences and this trend will decrease the efficiency of the system. The method is tested using genuine and forgery signature produced by 50 different persons. The highest accuracy rate achieved by our Devnagari handwritten signature recognition system was $96.12 \%$.

The proposed algorithm can be used as an effective signature verification system. The algorithm proposed was successfully made rotation invariant by the rotation of the image. The Equal Error Rate (EER) was found to be $31 \%$. It is the point where the value of the FAR and the FRR meet one another (shown in Fig. 5). The Equal Error Rate (EER) can further be improved by using better techniques for rotation, blurring and thinning. Using these algorithm random and simple forgeries can be easily detected. A great number of skilled forgeries can also be removed. It uses a compact and memory efficient storage of feature points which reduces memory overhead and results in faster comparisons of the data to be verified.

The overall experimental results are categorized in Table 3.3. However it is not sufficient to verify the validity of a signature only by comparing the physical image of it.

Table 3.3 : Overall Experimental Results

\begin{tabular}{|c|l|}
\hline $\begin{array}{c}\text { Percentage of } \\
\text { Similarity }\end{array}$ & \multicolumn{1}{c|}{ Result Displayed } \\
\hline $0-95 \%$ & $\begin{array}{l}\text { The sample signature is not } \\
\text { matching with database. }\end{array}$ \\
\hline $95-99 \%$ & $\begin{array}{l}\text { The sample signature } \\
\text { matches with the database. }\end{array}$ \\
\hline $99-100 \%$ & $\begin{array}{l}\text { The sample signature is the } \\
\text { original one. }\end{array}$ \\
\hline
\end{tabular}

\section{CONCLUSION}

Recognition of devnagari handwritten signature is a complex problem, which is not easily solvable. This work has presented a review of moment-based invariant functions, their history, basic principles, and methods how to construct them. We demonstrated that invariant functional can be used in image analysis as features for description and recognition of objects in degraded images. Invariant-based approach is a significant step towards robust and reliable object recognition methods. It has a deep practical impact because many pattern recognition problems would not be solvable otherwise. In practice, image acquisition is always degraded by unrecoverable errors and the knowledge of invariants with respect to these errors is a crucial point. This observation should influence future research directions and should be also incorporated in the education. To decrease the fluctuation of moment invariants, the image spatial resolution must be higher than the threshold of scaling and rotation. However, the resolution cannot be too high, because the computation will remarkably 
increase as the resolution increases. Therefore, the choice of resolution must balance computation and resolution on the real application. This approach can be used in multilingual character recognition as well.

The computation increases quickly as resolution increases. From the experimental studies, we find that the choice of image spatial resolution is very important to keep invariant features. To decrease the fluctuation of moment invariants, the image spatial resolution must be higher than the threshold of scaling and rotation. However, the resolution cannot be too high, because the computation will remarkably increase as the resolution increases. Therefore, the choice of resolution must balance computation and resolution on the real application.

Scope of Future Work : The concepts of Neural Networks as well as Hu's moment invariants hold a lot of promise in building systems with high accuracy. It can be extended for the recognition of words, sentence and documents. Another research interest will be on the character images degraded or blurred by various reasons. This approach can be used in multilingual character recognition as well.

\section{REFERENCES}

[1] Brijmohan Singh, Ankush Mittal and Debashis Ghosh, "An Evaluation of Different Feature Extractors and Classifiers for Offline Handwritten Devnagari Character Recognition", Journal of Pattern Recognition Research, pp. 269-277, 2011.

[2] Soumen Bag and Gaurav Harit, "Topographic Feature Extraction For Bengali And Hindi Character Images", International Journal of Signal \& Image Processing, Vol. 2, No. 2, pp. 181-196, June 2011.

[3] Bhupendra M. Chaudhari, Abhay B. Nehete, Kantilal P. Rane and Ulhas B. Shinde, "Efficient Feature Extraction Technique for Signature Recognition", International Journal of Advanced Engineering \& Application (IJAEA), pp. 64-70, January 2011 .

[4] Nan Xu, Li Cheng, Yan Guo, Xiaogang Wu and Jiali Zhao, "A Method for Online Signature Verification Based on Neural Network", IEEE Trans. of $3^{\text {rd }}$ International Conference on Communication Software and Networks (ICCSN), Wuhan, China, pp. 357-360, May 2011.

[5] Juan $\mathrm{Hu}$ and Youbin Chen, "Writer Independent Offline Handwritten Signature Verification based on Real Adaboost", IEEE Trans. of $2^{\text {nd }}$ International Conference on Artificial Intelligence, Management Science and Electronic Commerce (AIMSEC), pp. 6095-6098, August 2011.

[6] M. K. Hu, "Visual Pattern Recognition by Moment Invariants", IRE Trans. of Information Theory, Vol. 8, pp. 179-187, 1962.
[7] J. Flusser, "On the Independence of Rotation Moment Invariants", Journal of Pattern Recognition, Vol. 33, pp. 1405-1410, 2000.

[8] J. Flusser and T. Suk, "Rotation Moment Invariants for Recognition of Symmetric Objects", IEEE Trans. of Image Processing, Vol. 15, pp. 3784-3790, 2006.

[9] R. C. Gonzalez and R. E. Woods, "Digital Image Processing", $3^{\text {rd }}$ Edition, Prentice Hall of India, New Delhi, India, 2009.

\section{AUTHOR'S PROFILE:}

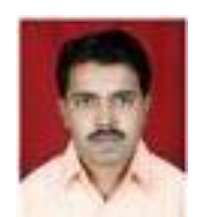

Shailendra Kumar Dewangan received B.E. in Electronics \& Telecommunication in year 2005 and M.E. in Communication Engineering from Shri Shankaracharya College of Engineering \& Technology (SSCET), Bhilai, Chhattisgarh, India. He is currently working as an Assistant Professor in the Department of Electronics \& Instrumentation Engineering at Chhatrapati Shivaji Institute of Technology (CSIT), Durg, Chhattisgarh, India. His areas of interest include Digital Signal Processing, information security, digital watermarking, advancements in communication technology, etc. He has published multiple articles in various International and National Journals \& Conferences. Besides he has lifetime membership of Indian Society of Technical Education (ISTE) and Associate membership of Institute of Electronics \& Telecommunication Engineers (IETE). 